\title{
Hadron production in deep inelastic lepton-nucleus scattering
}

\author{
A. Accardi ${ }^{1 *}$, V. Muccifora ${ }^{2 \dagger}$, H.J. Pirner ${ }^{13 \ddagger}$ \\ 1 Institut für Theoretische Physik der Universität Heidelberg \\ Philosophenweg 19, D-69120 Heidelberg, Germany \\ 2 INFN, Laboratori Nazionali di Frascati, I-00044 Frascati, Italy \\ 3 Max-Planck-Institut für Kernphysik, Heidelberg, Germany
}

\begin{abstract}
Predictions for semi-inclusive deep inelastic lepton-nucleus scattering are presented. Both the effects of gluon radiation by the struck quark and the absorption of the produced hadron are considered. The gluon radiation covers a larger window in virtuality $Q^{2}$ because of the increased deconfinement of quarks inside nuclei. The absorption of hadrons formed inside the nucleus is described with a flavor dependent cross section. Calculations for rescaled fragmentation functions and nuclear absorption are compared with the EMC and HERMES data for N, Cu and Kr targets with respect to the deuteron target. Predictions for Ne and Xe targets in the HERMES kinematic regime are given.
\end{abstract}

Keywords: DIS on nuclei, hadronization, modification of fragmentation functions.

PACS: 12.38.-t,13.60.Hb,13.60.Le

\footnotetext{
*E-mail address: accardi@tphys.uni-heidelberg.de

${ }^{\dagger}$ E-mail address: muccifora@lnf.infn.it

${ }^{\ddagger}$ E-mail address: pir@tphys.uni-heidelberg.de
} 


\section{Introduction}

The fragmentation of quarks into hadrons is still not completely understood. In fact, the final stages of hadronization involve low scale nonperturbative processes in QCD in Minkowski space together with hadronic wave functions which are not yet calculable. The nucleus may help to understand the space time evolution of a parton as the nucleons inside the nucleus play the role of very nearby detectors of the propagating object.

Deep inelastic lepton-nucleus scattering has the advantage that the lepton transfers a well-defined energy $\nu$ to the struck quark propagating through cold nuclear matter. Due to factorization in deep inelastic scattering (DIS), the semi-inclusive cross section can be described by the product of a parton distribution function (PDF) with a fragmentation function $(\mathrm{FF})$ and the hard scattering cross section. Fig. 1 shows a schematic diagram of semi-inclusive deep inelastic lepton scattering on a target, and the definitions of the four momenta of the particles involved in the process. In the naive parton model, the probability $q_{f}(x)$ that a quark of flavor $f$ with momentum fraction $x$ is present in the target is multiplied with the probability $D_{f}^{h}(z)$ that it hadronizes into a definite hadron $h$ which carries a momentum fraction $z$ of the quark.

While the inclusive deep inelastic scattering of high energy leptons on nuclei has been used to measure the quark distributions $q_{f}(x)$ in nuclei, semi-inclusive DIS (SIDIS) can be used to study the medium modifications of quark fragmentation functions $D_{f}^{h}(z)$. In SIDIS, besides the scattered lepton $l^{\prime}$, the leading hadron $h$ formed from the struck quark is detected with energy $E_{h}=z \nu$ in the target rest frame (see Table 1 for a list of kinematic variables). Experimentally, hadron multiplicities are obtained from normalizing the SIDIS yield $N^{h}$ to the DIS yield $N^{D I S}:\left(1 / N^{D I S}\right)\left(d N^{h}(x, z) / d z\right) \approx$ $\left(\sum_{f} e_{f}^{2} q_{f}(x) D_{f}^{h}(z) / \sum_{f} e_{f}^{2} q_{f}(x)\right)$, where the sum over all the quark of a flavor $f$ and charge $e_{f}$ is performed. It has been shown that, after integrating over a broad range of $x$, the multiplicities are a good approximation to the fragmentation functions (see Ref. [1] and references therein). Thus, a measurement of the hadron multiplicity in nuclei is a good tool to measure possible medium modifications of quark fragmentation functions.

The experimental results on semi-inclusive leptoproduction of hadrons from nuclei $[2,3]$ are usually presented in terms of multiplicity ratios $R_{M}^{h}$ between nuclear $(A)$ and deuteron $(D)$ targets, as functions of $z$ and $\nu$ respectively:

$$
\begin{aligned}
& R_{M}^{h}(z)=\frac{1}{N_{A}^{D I S}} \frac{d N_{A}^{h}}{d z} / \frac{1}{N_{D}^{D I S}} \frac{d N_{D}^{h}}{d z} \\
& R_{M}^{h}(\nu)=\frac{1}{N_{A}^{D I S}} \frac{d N_{A}^{h}}{d \nu} / \frac{1}{N_{D}^{D I S}} \frac{d N_{D}^{h}}{d \nu}
\end{aligned}
$$

In absence of nuclear effects, the ratio $R_{M}^{h}$ would be equal to 1 , and experimental results show that this is the case at high transfer energy $\nu[2]$.

The HERMES experiment [3] has brought new insight into the study of the hadronization process, since it has access to lower energy transfers $\nu$, where the hadronization is supposed to occur inside the nucleus. It has also extended the range of fractional momenta to larger values of $z$. In principle, a comparison with the EMC results [2], which involve much higher transfer energy, would allow us to separate the physics of gluon radiation from the absorption process of the produced hadron. 


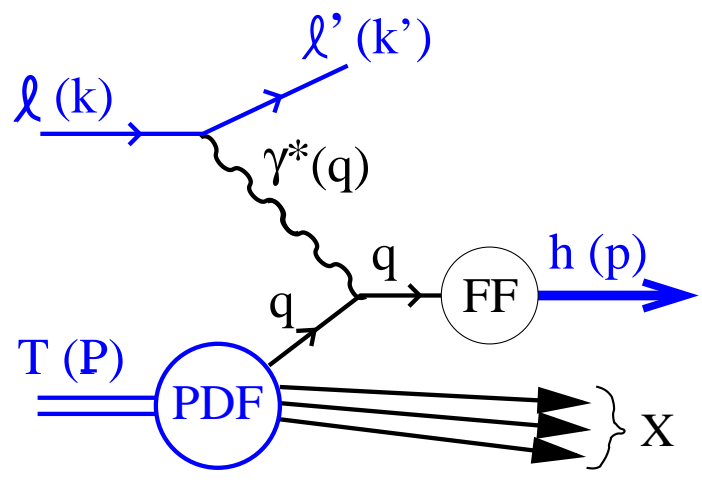

Four-momenta of the particles

\begin{tabular}{lrl}
\hline Particle & In lab. & frame \\
\hline Incident lepton & $k$ & $=(E, \vec{k})$ \\
Scattered lepton & $k^{\prime}$ & $=\left(E^{\prime}, \overrightarrow{k^{\prime}}\right)$ \\
Target nucleon/nucleus & $P$ & $=(M, \overrightarrow{0})$ \\
Virtual photon & $q$ & $=(\nu, \vec{q})$ \\
Produced hadron & $p$ & $=\left(E_{h}, \vec{p}\right)$ \\
\hline
\end{tabular}

Figure 1: Semi-inclusive hadron production in deep inelastic scattering on a target $\mathrm{T}$ in the pQCD factorization approach. Parton distribution functions (PDF) and fragmentation functions (FF) represent the non-perturbative input. Four-momenta of the particles involved in the process in the laboratory frame are defined in the table on the right.

There has been an intensive discussion on energy loss of partons in a cold and hot nuclear environment [5], which then leads to modified fragmentation functions in nuclei or in deconfined quark matter [6]. Recent data on high transverse momentum hadron produced in $\mathrm{Au}-\mathrm{Au}$ collisions at RHIC have truly opened up the domain of hard scattering and jet-quenching in heavy-ion collisions [7]. We would like to address this problem from a different perspective. Already in the vacuum, the struck quark loses substantial energy in the gluon cascade; the question arises how this cascade process is modified in the nuclear medium.

The goal of this paper is to describe the $R_{M}^{h}$ measurements $[2,3]^{1}$ in terms of modified fragmentation functions supplemented by nuclear absorption. We describe the modification of the structure function and fragmentation function in the framework of rescaling models $[8,9,11]$. These models have been successfully applied to the interpretation of nuclear structure functions. In this paper, we extend the rescaling model also to the medium modification of the quark fragmentation functions. For the nuclear absorption, we work out two specific fragmentation models based on work by Bialas-Gyulassy [12] and Bialas-Chmaj [13]. Applications of the above mentioned nuclear absorption models to DIS on nuclear targets may be found in Refs. [14] and [15].

Recently, other "QCD inspired" analysis such as the gluon-bremsstrahlung calculation for leading hadron production [16], the higher-twist pQCD computation of Ref. [17] and the effect of parton energy loss of Ref. [18] have been applied to hadron production in DIS on nuclei. Computations for meson production in DIS on nuclei in terms of stationary string model are also presented in Ref. [19].

The outline of the paper is as follows: in Sec. 2, we describe the modification of the fragmentation function by gluon radiation of the struck quark; in Sec. 3, we discuss the formation length and in Sec. 4, the nuclear absorption of the produced hadron. The comparison between the model predictions and the experimental data for charged hadrons is made in Sec. 5. The flavor dependence of the hadronization process is calculated and compared with data in Sec. 6. Conclusions are reported in Sec. 7.

\footnotetext{
${ }^{1}$ The SLAC data [4] will not be considered in this paper since the semi-inclusive distributions have been normalized to the target density rather than to the rate of inclusive events. Therefore they can not be directly compared to EMC and HERMES data.
} 
Kinematic variables

\begin{tabular}{rlll}
\hline Variable & Covariant & Lab. frame & \\
\hline$Q^{2}=-q^{2}$ & $\simeq 2 M x \nu$ & $\begin{array}{l}\text { Negative four-momentum squared } \\
\text { of the virtual photon. }\end{array}$ \\
$\nu=\frac{q \cdot p}{\sqrt{P^{2}}}=E^{\prime}-E$ & $\begin{array}{l}\text { Energy of the virtual photon in lab. } \\
\text { frame. }\end{array}$ \\
$x=\frac{-q^{2}}{2 P \cdot q}$ & $=\frac{Q^{2}}{2 M \nu}$ & $\begin{array}{l}\text { Bjorken scaling variable (fraction of } \\
P \text { carried by the struck parton in } \\
\text { the Breit frame) }\end{array}$ \\
$z=\frac{p \cdot P}{q \cdot P}$ & $=\frac{E_{h}}{\nu}$ & $\begin{array}{l}\text { Fraction of the virtual photon en- } \\
\text { ergy carried by the hadron. }\end{array}$ \\
$y=\frac{\nu \cdot P}{k \cdot P}$ & $\begin{array}{l}\text { Fraction of the incident lepton en- } \\
\text { ergy transferred to the target. } \\
\text { Invariant mass squared of the total } \\
\text { hadronic final state. }\end{array}$ \\
\hline
\end{tabular}

Table 1: Definitions of the kinematic variables used in the paper.

\section{Modification of parton distribution and fragmen- tation functions by gluon radiation.}

It has been shown (for a recent review see Ref. [20]), that a simple folding of nucleon structure functions with the momentum distribution of nucleons in the nucleus is insufficient to reproduce the nuclear structure functions. Nuclear structure and fragmentation functions certainly depend on the density of baryonic matter and on the amount of momentum sharing induced by the close packing of nucleons. A good description of nuclear structure functions has been achieved in the so-called deconfinement models, based on the hypothesis that quarks in bound nucleons have access to a larger region in space than in free nucleons [8-10].

Both parton distribution functions and fragmentation functions depend on the virtuality $Q^{2}$ of the DIS process. Their adjustment to the physical scale $Q^{2}$ takes into account all radiated gluons before and after the photon-quark interaction in the leading logarithm approximation. The long wavelength spectrum of gluons extends farther into the infrared toward low $Q^{2} \propto 1 / \lambda^{2}$ where $\lambda$ is the confinement scale. Deconfinement models assume a larger confinement scale $\lambda_{A}$ in nuclei, compared with the confinement scale $\lambda_{0}$ in free nucleons:

$$
\lambda_{A}>\lambda_{0} .
$$

Therefore, in nuclei gluon radiation would be affected by the assumed deconfinement of color.

Take, e.g., a parton distribution function and consider a quark which carries a momentum $Q_{0}$ when it is confined on a scale $\lambda_{0}$. If the scale changes to $\lambda_{A}$ it carries a corresponding momentum $Q_{A}$. If the free and bound nucleon would be characterized by these scales alone, the structure function of the bound nucleon would be related to the 
structure function of the free nucleon by replacing the measured $Q^{2}$ by $\left(\lambda_{A} / \lambda_{0}\right)^{2} Q^{2}$ in the arguments of the structure and fragmentation functions. Taking into account also the running of the QCD coupling, i.e. the existence of the QCD scale $\Lambda_{Q C D}$, the correct rescaling factor is $[9,10]$ :

$$
\xi_{A}\left(Q^{2}\right)=\left(\frac{\lambda_{A}}{\lambda_{0}}\right)^{2 \frac{\alpha_{s}}{\alpha_{s}\left(Q^{2}\right)}}
$$

where $\alpha_{s}\left(Q^{2}\right)$ is computed at leading order with $\Lambda_{\mathrm{QCD}}=200 \mathrm{MeV}$ and four quark flavours. The real scale factor $\xi_{A}\left(Q^{2}\right)$ considers that the low lying modes couple with a coupling constant $\bar{\alpha}_{s}$. Effectively, the DGLAP evolution of the nuclear structure function covers a larger interval in momentum compared with the corresponding functions in the nucleon at the same scale $Q$, and the nuclear modified distribution function $q_{f}^{A}$ for a quark of flavour $f$ reads:

$$
q_{f}^{A}\left(x, Q^{2}\right)=q_{f}\left(x, \xi_{A}\left(Q^{2}\right) Q^{2}\right)
$$

where $q_{f}$ is the corresponding distribution function in a free nucleon. For consistency it is necessary that partial deconfinement in nuclei does not only modify the parton distribution functions but also the fragmentation functions [11]. A procedure similar to the above one for the fragmentation function gives

$$
D_{f}^{h \mid A}\left(z, Q^{2}\right)=D_{f}^{h}\left(z, \xi_{A}\left(Q^{2}\right) Q^{2}\right),
$$

where $D_{f}^{h}$ is the fragmentation function of a quark of flavour $f$ into a hadron $h$ and $D_{f}^{h \mid A}$ is the nuclear modified fragmentation function. Of course such a simple adjustment of evolution scale is only a simple prescription to mimic the effects of the nucleus on the light-cone wave function of the bound nucleon. The rescaling relations (2.2) and (2.3) are expected approximately to be valid for $0.1<x<0.6$ and $z \geq 0.1$.

We use two models for the computation of the scale factor. The maximal deconfinement model (MD) $[8,10]$ assumes the onset of "colour conductivity" in nuclei and takes $\lambda_{A}=R_{A}$, where $R_{A}$ is the nuclear radius, so that

$$
\frac{\lambda_{A}}{\lambda_{0}}=\frac{R_{A}}{R_{p}}
$$

Since in this model the low lying modes can become very soft $\bar{\alpha}_{s}$ is frozen at $\bar{\alpha}_{s}=0.54$. The partial deconfinement model (PD) [9] assumes the deconfinement scale $\lambda_{A}$ to be proportional to the overlap of nucleons inside the given nucleus, and $\bar{\alpha}_{s}=\alpha_{s}\left(\mu_{A}^{2}\right)$, where $\mu_{A}=\frac{\lambda_{0}}{\lambda_{A}} \mu_{0}$ and $\mu_{0}=0.66 \mathrm{GeV}^{2}$ is the starting scale for DGLAP evolution in the free nucleon. A recent analysis [21], based on the experimental results [22] on the nucleon form factor in nuclei, found that the amount of deconfinement predicted by Ref. [9] is too large. In particular Ref. [21] gives an upper limit for $\left(\lambda_{A}-\lambda_{0}\right) / \lambda_{0} \sim 10 \%$ on iron, while Ref. [9] predicts a $15.3 \%$ enhancement. Therefore following Ref. [21] we reduce the $\lambda_{A}$ computed in Ref. [9] by a universal factor:

$$
\left(\frac{\lambda_{A}}{\lambda_{0}}-1\right)=\frac{0.10}{0.153}\left(\left.\frac{\lambda_{A}}{\lambda_{0}}\right|_{C J R R}-1\right) .
$$




\begin{tabular}{|c|c|ccccccc|}
\hline & & ${ }^{2} \mathrm{D}_{1}$ & ${ }^{14} \mathrm{~N}_{7}$ & ${ }^{20} \mathrm{Ne}_{10}$ & ${ }^{56} \mathrm{Fe}_{26}$ & ${ }^{63} \mathrm{Cu}_{29}$ & ${ }^{84} \mathrm{Kr}_{36}$ & ${ }^{131} \mathrm{Xe}_{54}$ \\
\hline \multirow{2}{*}{ PD model } & $\lambda_{A} / \lambda_{0}$ & 1.010 & 1.071 & 1.068 & 1.100 & 1.101 & 1.110 & 1.118 \\
& $\sqrt{\xi_{A}}(Q=1.5 \mathrm{GeV})$ & 1.014 & 1.110 & 1.106 & 1.164 & 1.165 & 1.182 & 1.195 \\
\hline \multirow{2}{*}{ MD model } & $\lambda_{A} / \lambda_{0}$ & 2.46 & 2.99 & 3.53 & 4.42 & 4.62 & 4.88 & 5.68 \\
& $\sqrt{\xi_{A}}(Q=1.5 \mathrm{GeV})$ & 3.65 & 4.82 & 6.13 & 8.47 & 9.02 & 9.76 & 12.3 \\
\hline
\end{tabular}

Table 2: Values of the deconfinement ratio $\lambda_{A} / \lambda_{0}$ and scale factors $\xi_{A}$ used in partial deconfinement (PD) model (see Eq. (2.5)) and maximal deconfinement (MD) model (see Eq. (2.4).

In Table 2 we show the values of the scale factor $\xi_{A}$ predicted by the aforementioned maximal deconfinement (MD) and partial deconfinement (PD) models for several nuclei.

The effect of the rescaling on the fragmentation function $D_{u}^{\pi^{+}}\left(z, \xi_{A} Q^{2}\right)[23]$ for different nuclei is shown in Fig. 2 both for the partial and maximal deconfinement models. Note that the MD model assumes a much larger scale factor, which results in a larger nuclear modification of hadron production especially at high $z$. One also sees that in the partial deconfinement model the effect of rescaling on the deuteron is much smaller than on heavy nuclei. This is different from the maximal deconfinement model, where only the nuclear radius matters and rescaling effects gradually vary between light and heavy nuclei. It is also worth to point out that the fragmentation function ratios shown in Fig. 2 increase in the low- $z$ region up to values larger than unity approximately at $z \sim 0.2$ for all nuclei in both rescaling models. This increase, common to all fragmentation functions with rescaled $Q^{2}$, comes from enhanced gluon radiation at small $z$ contributing to hadron production.
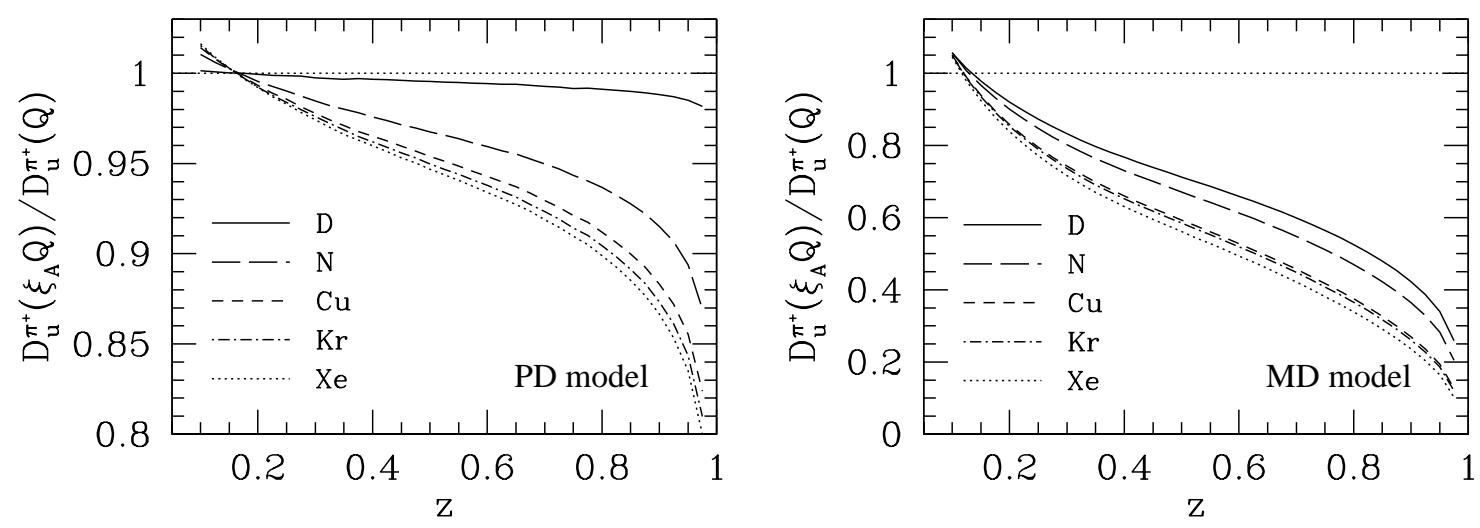

Figure 2: Ratio of the rescaled fragmentation functions $u \rightarrow \pi^{+}$to the standard ones at leading order [23] for $Q^{2}=2.25 \mathrm{GeV}^{2}$ as a function of $z$, in the case of partial deconfinement rescaling (PD) and maximal deconfinement rescaling (MD).

We calculate the multiplicity ratio of Eqs. (1.1) and (1.2) by using the rescaled PDF (2.2) and the rescaled FF (2.3) in the leading order pQCD computation of $N_{A}^{D I S}, d N_{A}^{h} / d z$ 
and $d N_{A}^{h} / d \nu$ :

$$
\begin{aligned}
\frac{1}{N_{A}^{D I S}} \frac{d N_{A}^{h}}{d z} & =\frac{1}{\sigma^{\ell A}} \int_{\text {exp. cuts }} d x d \nu \sum_{f} e_{f}^{2} q_{f}\left(x, \xi_{A} Q^{2}\right) \frac{d \sigma^{\ell q}}{d x d \nu} D_{f}^{h}\left(z, \xi_{A} Q^{2}\right) \\
\frac{1}{N_{A}^{D I S}} \frac{d N_{A}^{h}}{d \nu} & =\frac{1}{\sigma^{\ell A}} \int_{\text {exp. cuts }} d x d z \sum_{f} e_{f}^{2} q_{f}\left(x, \xi_{A} Q^{2}\right) \frac{d \sigma^{\ell q}}{d x d \nu} D_{f}^{h}\left(z, \xi_{A} Q^{2}\right) \\
\sigma^{\ell A} & =\int_{\text {exp. cuts }} d x d \nu \sum_{f} e_{f}^{2} q_{f}\left(x, \xi_{A} Q^{2}\right) \frac{d \sigma^{\ell q}}{d x d \nu} .
\end{aligned}
$$

Here $d \sigma^{\ell q} / d x d \nu$ is the differential cross-section for lepton-quark scattering computed at leading order (LO) in perturbation theory [24]:

$$
\frac{d \sigma^{\ell q}}{d x d \nu}=M x \frac{4 \pi \alpha^{2}\left(Q^{2}\right)}{Q^{4}}\left[1+(1-y)^{2}\right]
$$

Note that the experimental acceptance is explicitly accounted for in the integration limits in Eqs. (2.6)-(2.8). The experimental acceptances of the EMC and HERMES experiments are given in App. A. Nuclear isospin asymmetry is taken into account by using the averaged quark distribution function

$$
q_{f}^{A}=\frac{1}{A}\left[Z q_{f}^{p}+(A-Z) q_{f}^{n}\right] .
$$

The quark distribution function in the proton and in the neutron, respectively, $q_{f}^{p}$ and $q_{f}^{n}$, are related by isospin symmetry. In the numerical computations we use the leading order GRV98 parton distribution functions [25]. Since the PDF's appear both in the numerator and in the denominator of Eqs. (2.6)-(2.7), the theoretical uncertainty resulting from a different parametrization of the distribution functions is minimal. For the same reason, the effect of shadowing corrections to PDF's in the small-x region is negligible (the lowest $x$ for both EMC and HERMES experiments is $x \sim 0.02)$. We checked that by using the EKS98 parametrization [26] of PDF's with shadowing corrections, the multiplicity ratios (1.1) and (1.2) change by less than $1 \%$.

Furthermore, we analyzed charged hadron production with both the Khniel-KramerPötter parametrization of the FF at leading order (KKPLO) [27] and the leading order Kretzer's parametrization (KLO) [23]. The different parametrization of the FF produces a $1 \%$ difference in the multiplicity ratios. In the rest of the paper we shall use the KLO parametrization of the FF since it gives charge-separated fragmentation functions for pions and kaons, which are necessary for a comparison of the theoretical model with the HERMES data. Note, however, that by isospin symmetry arguments it is possible to relate charge-averaged FF's, like KKPLO, to charge-separated ones at the cost of an additional parameter [28].

Due to Lorentz dilatation, at large values of $\nu$ the hadrons are expected to form mainly outside the nucleus, so that the effect of reinteractions of the hadron with the nucleus are minimal. For this reason, the EMC data on hadron production in high energy DIS scattering on copper are ideal for a comparison of the partial and the maximal deconfinement model. The results for the $z$ - and $\nu$-dependence of the multiplicity ratio 

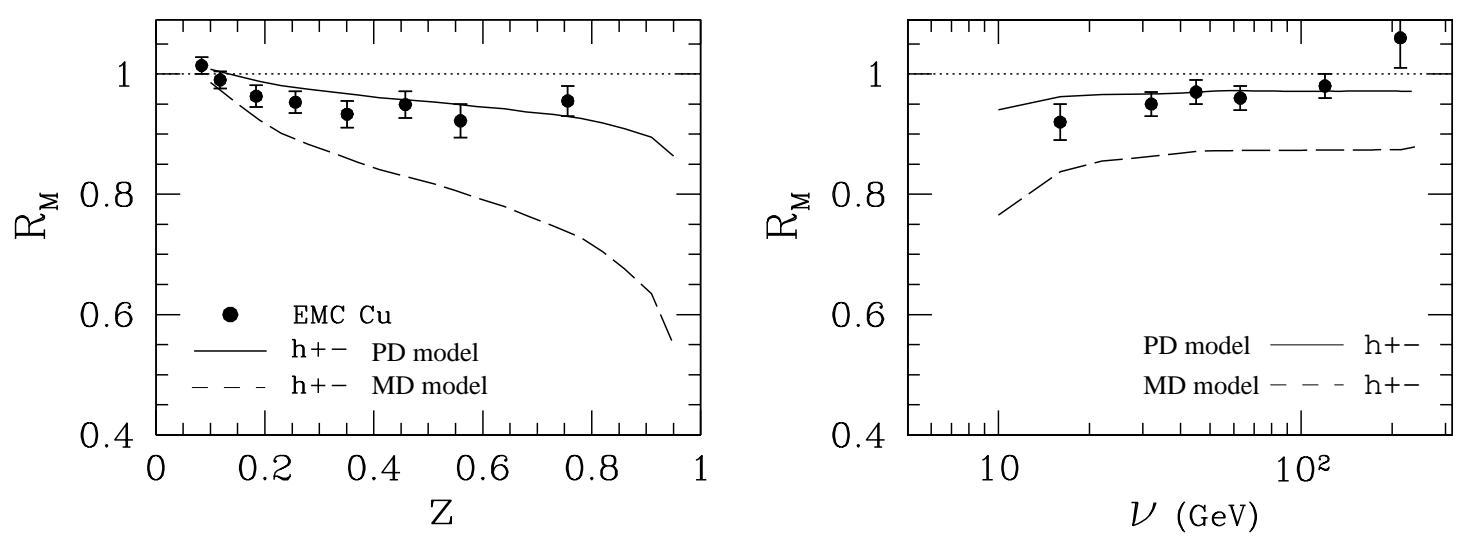

Figure 3: Theoretical multiplicity ratios $R_{M}$ as functions of $z$ and $\nu$ in the PD (solid lines) and MD (dashed lines) models are compared with EMC data [2] on $\mathrm{Cu}$. The theoretical curves between $10 \mathrm{GeV}<\nu<30 \mathrm{GeV}$ are computed with the experimental acceptance corresponding to a beam energy of $100 \mathrm{GeV}$ (see App. A).

are shown in Fig. 3 and compared with EMC data [2]. The maximal deconfinement model (dashed lines) underestimates the data over nearly the whole range in $z$ and $\nu$. On the contrary the partial deconfinement model (solid lines) better reproduces both the $z$ and $\nu$ experimental distributions but overestimates the data at low $\nu$ and $z$, thus suggesting that additional processes contribute in this region. As the maximal deconfinement model overpredicts the effect of deconfinement in the nucleus, in the following we shall consider only the partial deconfinement model.

\section{Formation length distribution in the Lund model}

The results presented in Fig. 3 show that the partial deconfinement rescaling model overestimates the multiplicity data slightly at small energy transfers $\nu$ and at small $z$. Therefore, we have to consider in addition the formation of the hadron and its subsequent interaction in the nuclear medium. As the hadron formation length decreases for lower $\nu$, the effect of nuclear interaction becomes more relevant in the kinematic region of the HERMES experiment, and the effect is amplified in a heavy target as the formation length is comparable with the nuclear radius. For a realistic calculation, a good understanding of the formation mechanism is necessary.

In this paper we follow the LUND model for the fragmentation process $[29,30]$ and we apply the hadron formation and interaction discussed in Ref. [12] to the DIS process. A picture of the space-time development of the fragmentation process in the Lund model is presented in Fig. 4. The process begins at $\mathrm{t}=0$ and $\mathrm{y}=0$ when the quark $q$ is ejected from a nucleon by the virtual photon. The quark propagates in the positive longitudinal direction $y$ and a colour string is formed between the quark and the target remnant. The string maps out the lightly shaded space time area. It breaks at points $C_{i}$ into smaller pieces due to quark-antiquark pair creation. When a quark moving in the positive direction meets an antiquark moving in the negative direction, they interact and form a so called "yo-yo" state, which is identified with a final state meson. This happens at points $P_{i}$ in 


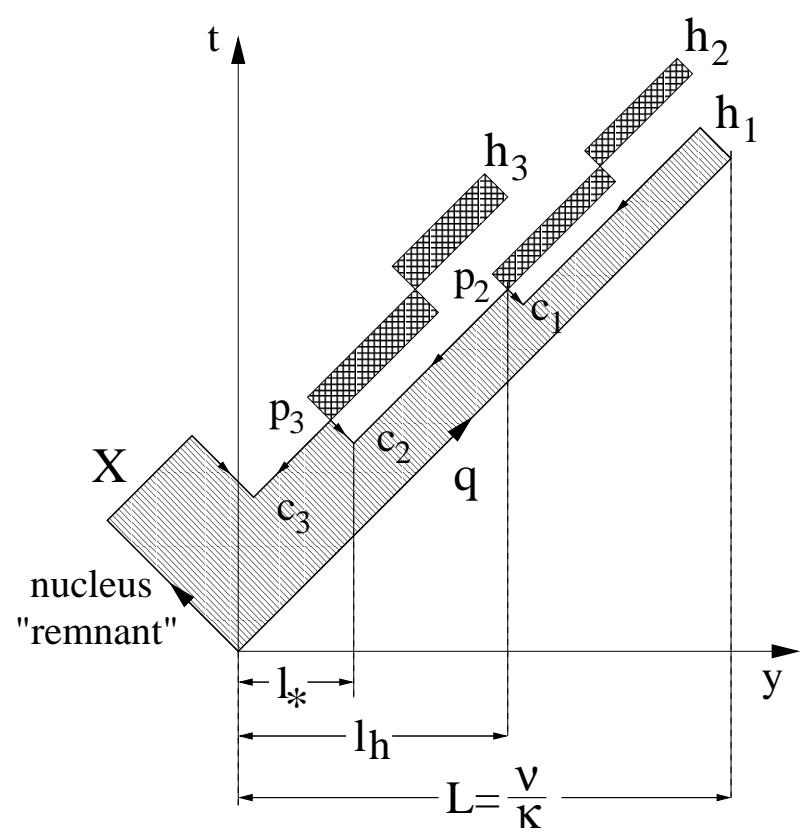

Figure 4: Formation lengths of the prehadron and hadron are shown in the Lund string model. The shaded area represents the world-sheet swept by the string. Produced hadrons, $h_{i}$, are ordered according to their rank $i$ (here $i=1,2,3$ and 3 hadrons are produced). At points $C_{i}$ prehadrons are created and at points $P_{i}$ the hadrons $h_{i}$ are formed. The formation lengths $l_{*}$ and $l_{h}$ at the bottom of the figure refer to the second-rank hadron $h_{2}$.

the figure, where the created hadron is denoted by $h_{i}$. We shall denote by $z_{i}$ the fraction of the energy it carries. Hadrons are ordered according to their rank $i$ [29]. Note that the first-rank hadron is always created at the end of the string, whose length is in our case

$$
L=\frac{\nu}{\kappa_{A}}
$$

where $\kappa_{A}$ is the string tension in the nuclear environment. If partial deconfinement occurs, also the string tension is rescaled in the nucleus:

$$
\kappa_{A} \lambda_{A}^{2}=\kappa \lambda_{0}^{2}
$$

where $\kappa \sim 1 \mathrm{GeV} / \mathrm{fm}$ is the string tension in the vacuum. Since the string tension is the physical quantity that sets the confinement scale, a larger confinement scale corresponds to a smaller string tension.

Let us focus our attention on the $h_{2}$ hadron in Fig. 4 . There are two relevant lengths for the fragmentation process:

(i) prehadron (or constituent) formation length $l_{*}$ at which the first constituent of $h_{2}$ is created.

(ii) the hadron (or yo-yo) formation length $l_{h} \leq L$ at which the hadron $h_{2}$ is formed.

In the Lund model these two lengths are related in the following way $[12,30]$ :

$$
l_{h}=l_{*}+z_{2} L
$$

At fixed $z$ they both increase linearly with the virtual photon energy $\nu$. However, as functions of $z$ they behave rather differently, especially at $z \rightarrow 1$, where $l_{*} \rightarrow 0$ and $l_{h} \rightarrow L$ (see Fig. 5). Therefore, we have two distinct scenarios of fragmentation, whether the prehadronic state interacts with the nucleus, or not. Analysis of the data on hadronnucleus and lepton-nucleus collisions strongly suggest that the prehadron behaves as an 


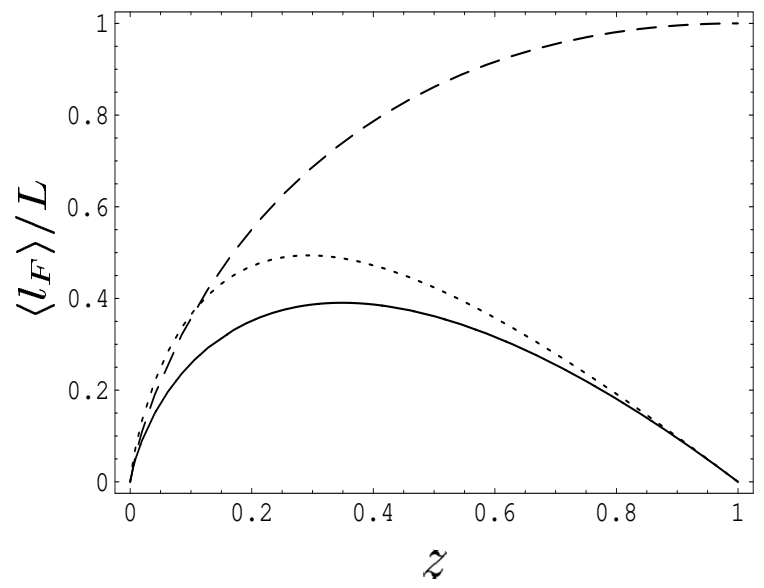

Figure 5: The average normalized prehadron formation length $\left\langle l_{*}\right\rangle / L$ computed in standard Lund model Eq. (3.4) with $C=0.3$ (solid line), compared with the one calculated from Eq. (3.5) used in the EMC analysis (dotted line). The normalized hadron (or yo-yo) formation length $\left\langle l_{h}\right\rangle / L$ is shown as a dashed curve.

hadron-like object long before final state hadrons actually appear [12,31]. Also an analysis of EMC and SLAC data confirms the importance of the prehadron interactions [15].

In App. $\mathrm{B}$ we derive the probability distribution $\mathcal{P}_{*}$ that a prehadronic state is formed at a formation length $y$ with momentum fraction $z$. In the case that the prehadron is composed of $q=u, d, s$ flavours, the distribution reads

$$
\begin{aligned}
\mathcal{P}_{*}(y ; z, L)= & \frac{z L}{y-z L}\left[\frac{y}{(y+z L)(1-z)}\right]^{C} \\
& \times\left\{\delta[y-(1-z) L]+\frac{1+C}{y-z L} \theta[(1-z) L-y]\right\} \theta[y]
\end{aligned}
$$

where $C \approx 0.3$ is the only parameter entering this expression. We can then compute the hadron average formation length $\left\langle l_{F}\right\rangle$, which we identify with the prehadronic formation length, $\left\langle l_{F}\right\rangle=\left\langle l_{*}\right\rangle(z, L)=\int d y y \mathcal{P}_{*}(y ; z, L)$ :

$$
\left\langle l_{F}\right\rangle=\left[1+\frac{1+C}{2+C} \frac{1-z}{z^{2+C}}{ }_{2} F_{1}\left(2+C, 2+C ; 3+C ; \frac{z-1}{z}\right)\right](1-z) z L,
$$

where ${ }_{2} F_{1}$ is the Gauss' hypergeometric function [32]. Note that as $z \rightarrow 1$ the average formation length $\left\langle l_{F}\right\rangle \rightarrow(1-z) \frac{\nu}{\kappa}$ behaves similarly to the formation length suggested by the gluon bremsstrahlung model of Ref. [16]. Indeed, quantum mechanics yields a large energy for an intermediate state consisting of a quark and a gluon with $z$ and $1-z$ momentum fractions when $z \rightarrow 1$. Consequently, if the hadron takes almost all the energy $(z \rightarrow 1)$ then the quark-gluon system is short lived and must hadronize almost immediately $[33]$.

In Fig. 5 we show the average formation length computed with Eq. (3.4) and $C=0.3$ as in the standard Lund model (solid line). This is the formula we use in the next sections. For comparison (dotted line) we show also the average formation length used in the analysis of the EMC data [34],

$$
\left\langle l_{F}\right\rangle=\left[\frac{\ln \left(1 / z^{2}\right)-1+z^{2}}{1-z^{2}}\right] z L,
$$




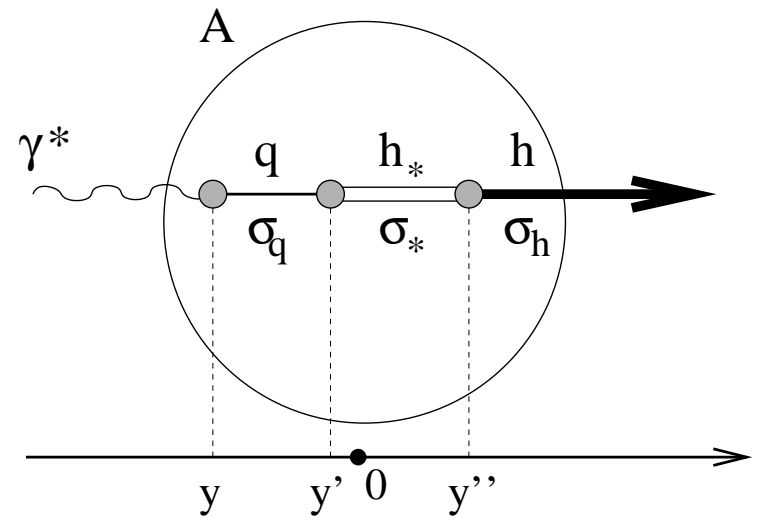

Figure 6: Nuclear attenuation of a hadron $h$ : the virtual photon $\gamma^{*}$ interacts with a quark $q$ at a longitudinal coordinate $y$; the quark turns into a "prehadronic" state $h_{*}$ at position $y^{\prime}$ and the hadron $h$ is formed at $y^{\prime \prime}$. Each state interacts with the surrounding nucleons with a cross-section $\sigma_{q}, \sigma_{*}$ and $\sigma_{h}$, respectively.

which overestimates up to $30 \%$ the Lund model result. The above formation length is obtained in the Lund model with a non-standard choice of parameters, as described in App. B. Finally, the contribution of the hadron (or yo-yo) formation lenght $l_{h}$ in the standard Lund model is shown with a dashed curve.

\section{Absorption of the produced hadron in the nucleus}

The spatial evolution of a quark $q$ created by the virtual photon $\gamma^{*}$ at a longitudinal position $y$ is shown in Fig. 6 in all intermediate stages. In the first stage the quark propagates to the position $y^{\prime}>y$ where a prehadronic state $h_{*}$ is formed. In the second stage the final hadron $h$ is created at the point $y^{\prime \prime}>y^{\prime}$.

The quark, the prehadronic state and the final hadron propagate through nuclear matter and interact with the surrounding nucleons. As a result, each of the three states may undergo inelastic interactions and/or lose longitudinal energy through elastic rescatterings. In a first approximation we may assume that the final hadron with a value of $z$ corresponding to the fragmentation process in vacuum will be observed provided that none of the three propagating states has interacted with the nucleus. By defining the nuclear absorption factor $\mathcal{N}_{A}(z, \nu)$ as the probability that neither the $q, h_{*}$, nor $h$ have interacted with a nucleon, the multiplicity ratios of Eqs. (2.6) and (2.7) are modified as follows:

$$
\begin{aligned}
& \frac{1}{N_{A}^{D I S}} \frac{d N_{A}^{h}}{d z}=\frac{1}{\sigma^{\ell A}} \int_{\text {exp. cuts }} d x d \nu \sum_{f} e_{f}^{2} q_{f}\left(x, \xi_{A} Q^{2}\right) \frac{d \sigma^{\ell q}}{d x d \nu} D_{f}^{h}\left(z, \xi_{A} Q^{2}\right) \mathcal{N}_{A}(z, \nu), \\
& \frac{1}{N_{A}^{D I S}} \frac{d N_{A}^{h}}{d \nu}=\frac{1}{\sigma^{\ell A}} \int_{\text {exp. cuts }} d x d z \sum_{f} e_{f}^{2} q_{f}\left(x, \xi_{A} Q^{2}\right) \frac{d \sigma^{\ell q}}{d x d \nu} D_{f}^{h}\left(z, \xi_{A} Q^{2}\right) \mathcal{N}_{A}(z, \nu) .
\end{aligned}
$$

While the fragmentation functions $D_{f}^{h}$ are sensitive to the virtuality $Q^{2}$ of the fragmentation process in the medium, the nuclear absorption factor $\mathcal{N}_{A}$, which depends on $z$ and $\nu$, is sensitive to the hadron energy in the rest frame of the nucleus.

We will investigate two models for the computation of the nuclear absorption factor: the Bialas-Gyulassy (BG) model, [12], and the Bialas-Chmaj (BC) model, [13]. These models require the probability distribution function of the hadron formation length and the average formation length discussed and derived in Sec. 3. In order to reduce the number of the parameters of these models we set the quark-nucleon cross-section $\sigma_{q}=0$, 
since the analysis of the DIS data on $R_{M}^{h}$ reported in $[2,3,34]$ does not indicate a significant interaction of the quark with the nuclear medium. Moreover, we do not give the formulae for a separate evolution of the prehadron to the full hadronic size in the main text. Then, the prehadronic state $h_{*}$ and the hadron $h$ are treated effectively as a single object $h_{*}=h$ created at $y^{\prime}$ which propagates in the nucleus with a single cross-section $\sigma_{*}=\sigma_{h}$. The effect of distinguishing the prehadronic state by a different cross section $\sigma_{*} \neq \sigma_{h}$ is discussed in Sec. 5 and 6, and the formulae needed in the computations of the absorption effect are discussed in App.C.

\subsection{Bialas-Gyulassy (BG) model}

In the Bialas-Gyulassy (BG) model [12] the nuclear absorption for a one-step fragmentation process with $\sigma_{*}=\sigma_{h}$ depends on three parts: the nuclear density profile $\rho_{A}(b, y)$ normalized to unity as a function of impact parameter $b$ and longitudinal coordinate $y$; the probability distribution $\mathcal{P}_{*}\left(y^{\prime}-y, z, L\right)$ that the prehadron is formed after the length $y^{\prime}-y$; and the probability $\left[W_{0}\left(y^{\prime}\right)\right]^{A-1}$ that the hadron created at $y^{\prime}$ does not interact with the nucleus. The nuclear absorption factor has the following form:

$$
\mathcal{N}_{A}=\int d^{2} b \int_{-\infty}^{\infty} d y \rho_{A}(b, y) \int_{y}^{\infty} d y^{\prime} \mathcal{P}_{*}\left(y^{\prime}-y, z, L\right)\left[W_{0}\left(y^{\prime}\right)\right]^{A-1}
$$

where

$$
W_{0}\left(y^{\prime}\right)=1-\sigma_{h} \int_{y^{\prime}}^{\infty} d y_{*} \rho_{A}\left(b, y_{*}\right) .
$$

For $\mathcal{P}_{*}$ we will use the probability distribution (3.3) discussed in Sec. 3 .

\subsection{Bialas-Chmaj (BC) model}

The Bialas-Chmaj (BC) model [13] is a simpler version of the BG-model for including nuclear absorption. In the $\mathrm{BC}$ model the nuclear absorption factor depends on the probability $P_{h}\left(y^{\prime}, y\right)$ that the hadron $h_{*}=h$ is formed at a distance $y^{\prime}-y$ from the $\gamma^{*} q$ interaction point,

$$
P_{h}\left(y^{\prime}-y\right)=1-e^{-\left(y^{\prime}-y\right) /\left\langle l_{F}\right\rangle},
$$

and on the survival probability $S_{A}(b, y)^{A-1}$ of this hadron, where for a one step fragmentation process with $\sigma_{*}=\sigma_{h}$ one has

$$
S_{A}(b, y)=1-\sigma_{h} \int_{y}^{\infty} d y^{\prime} P_{h}\left(y^{\prime}-y\right) \rho_{A}\left(b, y^{\prime}\right) .
$$

The nuclear absorption factor is then written as:

$$
\mathcal{N}_{A}=\int d^{2} b \int_{-\infty}^{\infty} d y \rho_{A}(b, y)\left[S_{A}(b, y)\right]^{A-1}
$$

where the average formation length $\left\langle l_{F}\right\rangle=\left\langle l_{F}\right\rangle(z, \nu)$ we will use has been derived in Sec. 3, Eq. (3.4). 


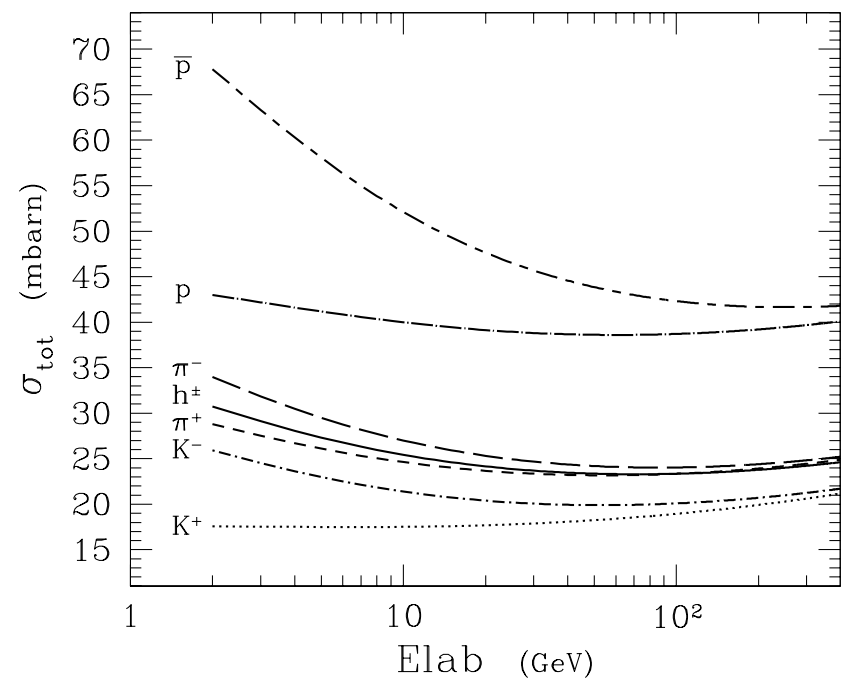

Figure 7: Isospin averaged hadronnucleon total cross section versus the hadron energy $E_{h}=E_{l a b}$, in the case of krypton nucleus, for positive and negative pions and kaons, and protons and antiprotons. The charged hadron cross-section $\sigma_{h}$ has been computed as weighted sum of individual cross sections for pions, kaons, protons and antiprotons.

To understand the BC model and its relationship to the BG model, Eq. (4.3), we can approximate $\rho_{A}(y, b)$ with a uniform nuclear density $\rho_{A}(y, b)=\rho_{0} / A$ inside a hard sphere. Here $\rho_{0}$ is the nuclear matter density. In this approximation the integrations in Eq. (4.6) may be carried out explicitly and yield

$$
\mathcal{N}_{A}^{(B C)}=\left[1-\frac{\sigma_{h} \rho_{0}}{A}\left\{R(b)-\left(y-\left\langle l_{F}^{\mathrm{e} f f}\right\rangle\right) \theta(R(b)-y) \theta(R-b)\right\}\right]^{A-1} .
$$

Here $R(b)^{2}=\sqrt{R^{2}-b^{2}}$ and the effective formation length $\left\langle l_{F}^{\text {eff }}\right\rangle$ is defined as:

$$
\left\langle l_{F}^{\mathrm{eff}}\right\rangle=\left\langle l_{F}\right\rangle\left[1-e^{-(R(b)-y) /\left\langle l_{F}\right\rangle}\right] .
$$

It is then easy to see that Eq. (4.7) can be derived from the Bialas-Gyulassy formula (4.3) if we choose

$$
\mathcal{P}_{*}\left(y^{\prime}-y\right)=\delta\left[\left(y^{\prime}-y\right)-\left\langle l_{F}^{\mathrm{eff}}\right\rangle\right] .
$$

In this sense, the BC model for nuclear absorption is an effective model for absorption which describes the fragmentation process by modifying the average formation length according to Eq. (4.8), and letting the hadron to be formed suddenly after a well defined formation length, instead of assuming a probability distribution in formation lengths. Nonetheless, as we shall discuss in Sec. 5 and Sec. 6, the BC model gives a useful phenomenological description of nuclear absorption processes.

\section{$5 \quad$ Numerical results for multiplicity ratios in nuclei}

In this section we discuss the results of our model for semi-inclusive charged hadron production in nuclei including effects of perturbative gluon radiation and absorption, and compare them to EMC and HERMES experimental data.

In the computation of the nuclear absorption factor, we use as a density for the deuteron the sum of the Reid's soft-core S- and D-wave functions squared [35]. For heavier nuclei we use a Woods-Saxon density with radius $R_{A}=1.12 A^{1 / 3}-0.86 A^{-1 / 3} \mathrm{fm}$. 
As discussed in Sec. 4, the fragmentation of the quark is considered without differentiation between the prehadron-nucleon and hadron-nucleon cross sections. For the hadronnucleon cross sections, we take the isospin averaged hadron-proton $\sigma_{h p}$ and hadronneutron $\sigma_{h n}$ total cross sections:

$$
\sigma_{h}\left(E_{h}\right)=\frac{1}{A}\left[Z \sigma_{h p}\left(E_{h}\right)+(A-Z) \sigma_{h n}\left(E_{h}\right)\right] .
$$

As the total hadron-nucleon cross sections include also the elastic ones, the corresponding results represent an upper limit with respect to the nuclear absorption effect in the multiplicity ratios. The energy dependence of $\sigma_{h p}$ and $\sigma_{h n}$ [36] has been explicitly taken into account, and is at variance with respect to the original models $[12,13]$. The energy behavior of the total hadron-nucleon cross section is shown in Fig. 7, in the case of a krypton target, for positive/negative pions and kaons. The corresponding charged hadron cross section has been derived as weighted sum of individual cross sections for pions, kaons, protons and antiprotons. The weighted sum has been performed by taking into account the measured relative yield of various hadrons reported by the HERMES experiment in the $3-23 \mathrm{GeV}$ region [3] as this region is very sensitive to the variation of the hadron-nucleon cross section.

As the model predictions are sensitive to the average kinematic variables used in the computations, in Fig. 8 we compare our LO theoretical computations of the average $\nu$, $z$ and $Q^{2}$ with the corresponding experimental values. The model reproduces reasonably well the experimental $\left\langle Q^{2}\right\rangle,\langle z\rangle$ and $\langle\nu\rangle$ behaviour. The step structures found in the LO computation of $\langle\nu\rangle(z)$ and $\langle z\rangle(\nu)$ are due to the experimental kinematic cuts shown in Fig. 12 of App. A. The differences compared with the experimentally observed smoother dependence of $\langle\nu\rangle$ and $\langle z\rangle$ may come from NLO corrections or higher-twist terms. Therefore, in the computation of the multiplicity ratios we replaced the arguments of Eq. (4.1) by experimental values when available:

$$
\begin{aligned}
q_{f}\left(x, \xi_{A} Q^{2}\right) & \longrightarrow q_{f}\left(x, \xi_{A}\left\langle Q^{2}\right\rangle_{\exp }(z)\right) \\
D_{f}^{h}\left(z, \xi_{A} Q^{2}\right) & \longrightarrow D_{f}^{h}\left(z, \xi_{A}\left\langle Q^{2}\right\rangle_{\exp }(z)\right) \\
\mathcal{N}_{A}(z, \nu) & \longrightarrow \mathcal{N}_{A}\left(z,\langle\nu\rangle_{\exp }(z)\right)
\end{aligned}
$$

and in Eq. (4.2)

$$
\begin{aligned}
q_{f}\left(x, \xi_{A} Q^{2}\right) & \longrightarrow q_{f}\left(x, \xi_{A}\left\langle Q^{2}\right\rangle_{\exp }(\nu)\right) \\
D_{f}^{h}\left(z, \xi_{A} Q^{2}\right) & \longrightarrow D_{f}^{h}\left(z, \xi_{A}\left\langle Q^{2}\right\rangle_{\exp }(\nu)\right) \\
\mathcal{N}_{A}(z, \nu) & \longrightarrow \mathcal{N}_{A}\left(\langle z\rangle_{\exp }(\nu), \nu\right)
\end{aligned}
$$

The effect of using experimental averages instead of the LO-computed ones by Eqs. (4.1) and Eq. (4.2), maximally yields $2-3 \%$ variation in the presented multiplicity ratios. The multiplicity ratios for positive and negative charged hadrons are calculated by using the fragmentation functions for $h^{ \pm}$in Kretzer's parameterization, with the meson formation time in the Lund model, Eqs. (3.3) and (3.4), and with the average cross-section shown in Fig. 7. Since charged hadrons are dominated by meson production, this is a good approximation to the sum of individual hadron fragmentation yields multiplied by individual absorption factors. In Sec. 6 we will also calculate multiplicity ratios for pions and kaons 

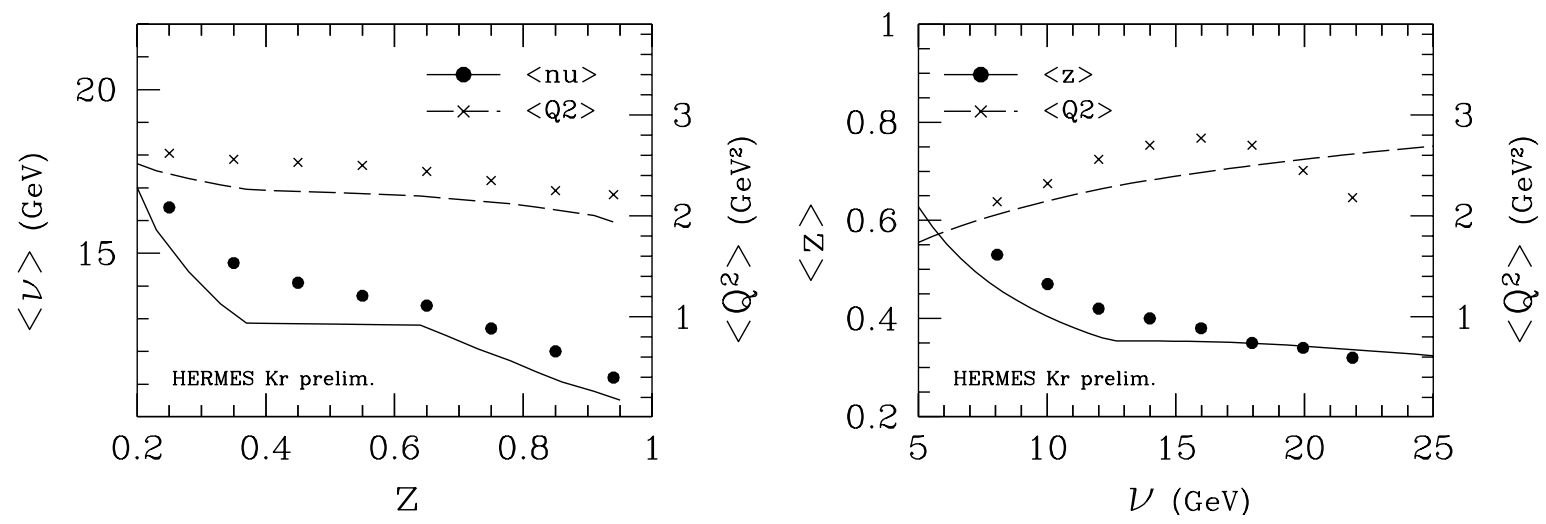

Figure 8: Average theoretical values of kinematic variables computed with the LO formulae (2.6) and (2.7) are compared with the experimental kinematic variables at HERMES for $\pi$ and $K$ production on $\operatorname{Kr}$ [37]. Left: $\langle\nu\rangle$ and $\left\langle Q^{2}\right\rangle$ as a function of $z$. Right: $\langle z\rangle$ and $\left\langle Q^{2}\right\rangle$ as a function of $\nu$.

separately. We leave out an extra calculation of baryon and antibaryon production, since such a calculation would require to know the contribution of target fragmentation and to extract separate fragmentation functions for $p$ and $\bar{p}$ from the charge averaged KKP parameterization. Furthermore a different formation mechanism is possible for baryons or antibaryons, see Eqs. (B.15) and (B.16).

The theoretical calculations of the multiplicity ratio for charged hadrons are presented in Fig. 9 and compared with the EMC and HERMES data for different nuclei. In the figure, the three different theoretical curves correspond to calculations with rescaled fragmentation functions alone (dashed curve), with rescaling plus nuclear absorption in the BC model (solid curve), and with rescaling plus absorption in the BG model (dotted curve).

In the case of BG absorption with the probability distribution of Eq. (3.3), the general shape of the curve deviates from the experimental behavior and the calculation underestimates the multiplicity ratio both at small and large $z$. In the case of BC absorption there is a better agreement with the experimental behaviour. Note that in this case we used the average formation length of Eq. (3.4) where we fixed the effective string tension $\kappa_{B C}=0.4 \mathrm{GeV} / \mathrm{fm}$ in order to agree with the experimental data. As already observed in Refs. [2,34] and [38] the Bialas-Chmaj model with $\kappa=1 \mathrm{GeV} / \mathrm{fm}$ overestimates by far the absorption effects, while, with a reduced effective string tension, a reasonably good description of the data is obtained inside the statistical and systematic errors.

The observed differences between the data and the model predictions in the low- $z$ region can be interpreted in several ways. In the described models for hadron absorption, a hadron which interacted with a nucleon is simply removed without accounting for a possible lowering of $z$ due to the interaction. A modification of the string fragmentation mechanism to take this into account was proposed in Ref. [14]. Moreover, the differences at low $z$ can be ascribed to target fragmentation protons, which can give a significant contribution to the multiplicity in this region. Also the effect of different prehadron and hadron cross sections $\sigma_{*} \neq \sigma_{h}$ may play a role. This has been investigated by fixing the prehadronic state cross-section $\sigma_{*}=0.5 \sigma_{h}$. The corresponding results, presented in the bottom right plot of Fig. 9, show an enhanced multiplicity ratio, but the shape of 

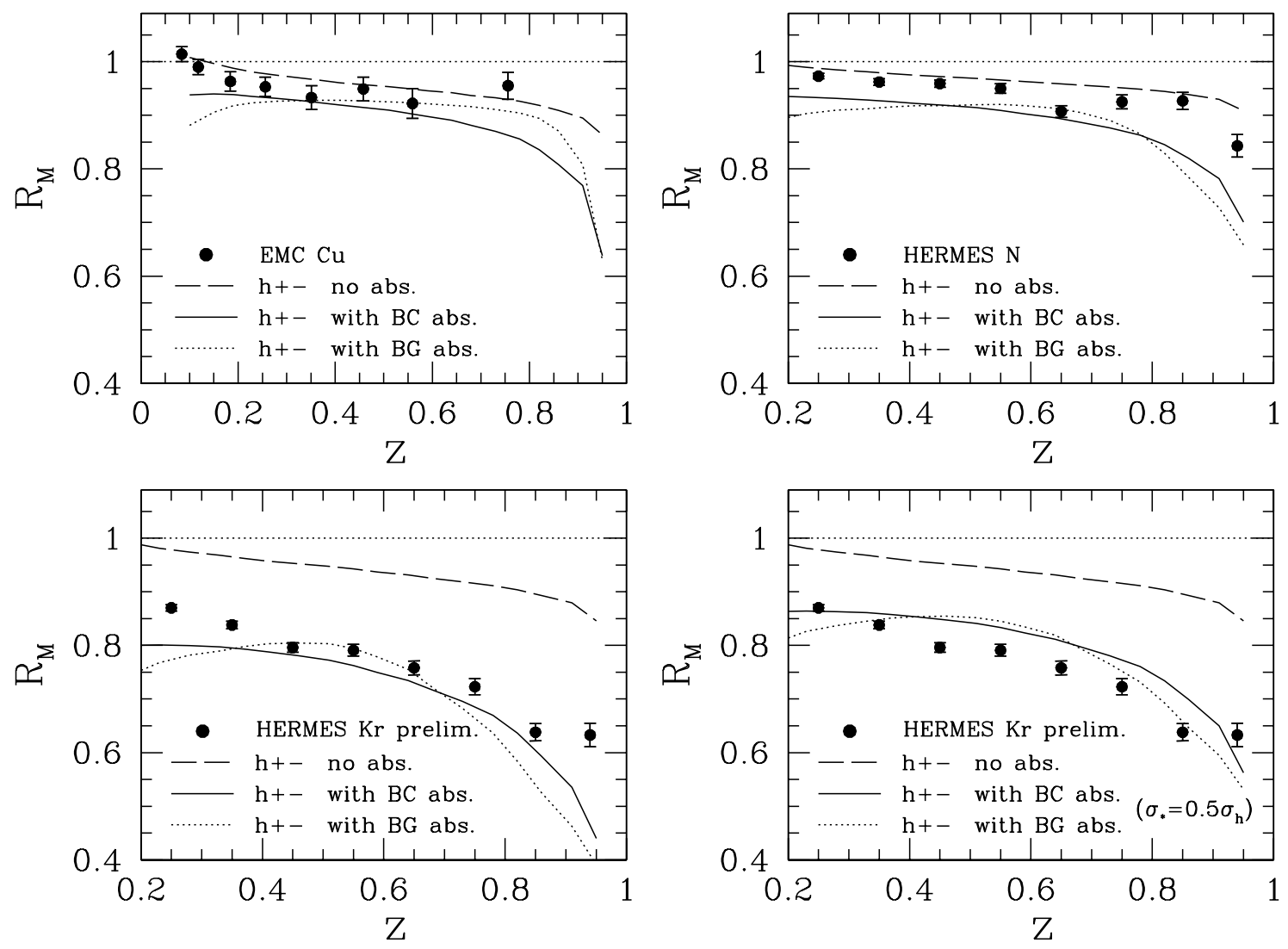

Figure 9: Theoretical multiplicity ratios of charged hadrons for $\mathrm{Cu}$ (EMC), Kr and N (HERMES) targets as function of $z$ compared with the data $[2,3]$. Dashed lines show the predictions without absorption and only rescaling, according to Eqs. (2.6)-Eqs. (2.8); solid lines give the calculation with rescaling and absorption according to Eqs. (4.6) and (3.4) for the BC model; dotted lines with rescaling plus absorption according to Eq. (4.3) and (3.3) for the BG model. In the bottom right plot, the solid and dotted curves represent the result of the computations with $\sigma_{*}=0.5 \sigma_{h}$. The error bars represent the statistical uncertainty only. Systematic uncertainties for the HERMES nitrogen and krypton data are $3 \%$ and $3.5 \%$ respectively $[3,37]$.

the $\mathrm{BG}$ predictions still disagrees with the data and the $\mathrm{BC}$ predictions overestimate the experimental results. Therefore, we will use the $\mathrm{BC}$ model with prehadronic cross section equal to the hadronic cross section to predict $R_{M}^{h}$ in the region $0.2 \lesssim z \lesssim 0.9$ also for different fragmentation products and for different targets (see Sec. 6).

As we can see in Fig. 9, the effect of the absorption is less important at EMC in $\mathrm{Cu}$ than the absorption effect at HERMES in $\mathrm{Kr}$, which is a nucleus of similar size, due to the larger energy transfer $\nu$ in the EMC data $(\langle\nu\rangle=62 \mathrm{GeV})$ compared to HERMES $(\langle\nu\rangle \sim 12 \mathrm{GeV})$. In the HERMES kinematic range both rescaling and absorption contribute. Absorption becomes the dominant effect in $\mathrm{Kr}$ tending to mask the rescaling effect. In the case of the $\mathrm{N}$ nucleus the theoretical result underestimates the experimental data. This may be due to an overestimate of the rescaling effect in light nuclei, which have a large surface to volume ratio.

It is worth to point out that the accuracy of the HERMES data may distinguish different models for the space-time development of the fragmentation functions. The main tool in this respect is the availability of data at large $z$, for both light and heavy 
nuclei, and for different hadrons. This combination explores the fragmentation process in a region which has not been investigated before. Obviously, our model has problems for $z>0.9$ to explain the data. For such large $z \mathrm{~L}$, higher-twist processes in fragmentation, like the direct ejection of preformed pions in the nucleon, may be important. We think in other regions the data do not give a specific hint on the importance of higher-twist effects. Also it may be interesting to extend the predictions of other models of fragmentation to the full data set.

\section{Charge and flavour dependence}

The complete particle identification of the HERMES experiment allows us to disentangle the information for different hadron types. Recently, the HERMES collaboration reported measurement of $R_{M}^{h}$ for pions, kaons, protons and antiprotons where the medium effects on $\pi^{+}$and $\pi^{-}$are equal, while significant differences are observed between $K^{+}$and $K^{-}$ and especially between $p$ and $\bar{p}$. The results for individual hadron species may reveal differences in the modification of $q$ and $\bar{q}$ fragmentation functions as also suggested in Ref. [17], in the formation time of baryons and mesons [3,33], or in the size of the hadronic and prehadronic cross sections.

In the following, we present the model results at the HERMES kinematics for charged pions and kaons, as the present version of this model does not apply to baryon production. The predictions are presented in Fig. 10, and compared with the HERMES data, both as a function of $\nu$ and $z$. In each plot we show the contribution of the rescaling and the total contribution of rescaling and absorption for both charged states. One sees that the average charged meson spectra $\frac{1}{2}\left(\pi^{+}+\pi^{-}\right)$and $\frac{1}{2}\left(K^{+}+K^{-}\right)$are reduced by the rescaling of the singlet fragmentation functions, which are strongly affected by the anomalous dimension of gluon splitting. At large $z$, the observed $\sim 10 \%$ effect can be calculated analytically. The difference of $\left(\pi^{-}-\pi^{+}\right)$and $\left(K^{-}-K^{+}\right)$spectra is governed by the non-singlet fragmentation function, which is weighted with the excess of neutron number $(\mathrm{N}=48)$ compared with the proton number $(\mathrm{Z}=36)$ in krypton. This explain the slight enhancement of $\pi^{-}$and $K^{-}$spectra at large $z$ in the rescaling model shown in Fig. 10 (upper curves in each plot). Subsequent absorption with a larger nuclear cross sections for $\pi^{-}$and $K^{-}$(see Fig. 7) corrects this difference.

The data for $\pi^{+}$and $\pi^{-}$production indeed show no difference in the multiplicity ratio, and are in nice agreement with the model predictions. We do not show calculations of $\pi^{0}$ mesons since they are very similar to the curve for charged pions. It is worth to point out that there is a nice agreement between model predictions and pion data also in the low $z$ region. This finding suggests that the observed difference in the spectra of charged hadrons (see Fig. 9) should be ascribed to proton production from target fragmentation which has not been considered theoretically. It is also possible to disentangle the contributions of the prehadronic and hadronic absorption by setting $\sigma_{h}=0$ and $\sigma_{*}=\sigma_{\pi}$ in the calculation of $R_{M}^{\pi}$. The resulting $z$-distribution of charged pions is shown as dotted curve in the upper left plot of Fig. 10. The neglect of hadronic absorption leads to a very small increase of $R_{M}^{\pi}$ at small $z$. This result shows that in the HERMES kinematic region mainly the prehadron absorption contributes because the hadron average formation length $\left\langle l_{h}\right\rangle=\left\langle l_{F}\right\rangle+\frac{z \nu}{k_{A}}$ is larger than the size of the krypton nucleus. 

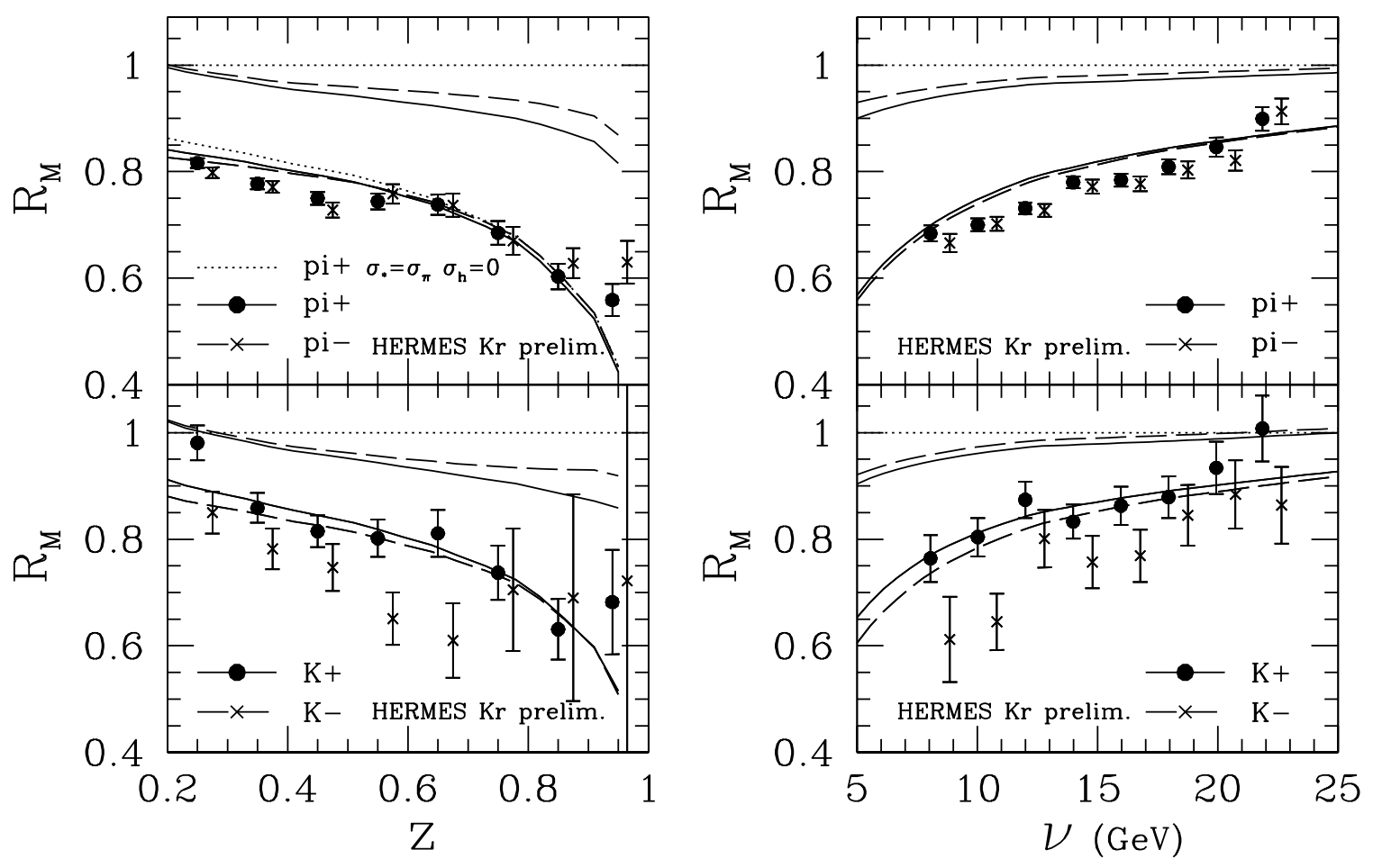

Figure 10: Charge- and flavour-separated theoretical multiplicity ratios $R_{M}(\nu)$ and $R_{M}(z)$, compared with preliminary HERMES data on Krypton target [37] (the data for negatively charged hadrons have been slightly shifted to the right to improve the readability of the figure). The upper pair of curves includes rescaling without absorption for positive and negative particles, and the lower pair rescaling plus BC absorption for positive and negative particles. The dotted curve in the upper left plot show the result by setting $\sigma_{h}=0$ and $\sigma_{*}=\sigma_{\pi}$ in the calculation of $R_{M}^{\pi}$. The error bars represent the statistical uncertainty only. Systematic uncertainty is about $4 \%$ [37].

For $K^{+}$production there is also a general agreement between data and model computations, while experimental results point to a stronger than calculated absorption of $K^{-}$ mesons. We found that an increase of $\sigma_{*}$ of at least $50 \%$ would be necessary in order to reproduce the experimental data for $K^{-}$. The discrepancy between theory and data may also point to a different formation mechanism for the negative kaons, as they do not contain any nucleon valence quarks, which dominate in the HERMES kinematics. This may imply a shorter formation length than predicted by Eq. (3.4), hence a larger absorption, since rank 1 hadrons would not participate in the $K^{-}$formation, see Eq. (B.16).

The forthcoming HERMES data for different hadron types on light and heavy nuclei may help to further disentangle rescaling and absorption effects and to clarify details of the fragmentation process. In Fig. 11 we show the predictions of the model for $\mathrm{Ne}$ and Xe nuclei in the HERMES kinematic region for charged pions and kaons. To this respect, the absorption mechanism can be studied on heavy nuclei, while light nuclei are more sensitive to the rescaling effect alone. One characteristic feature of the rescaling corrections is the increase of the multiplicity ratio $R_{M}>1$ for $z<0.2$ which in the case of $K^{+}, K^{-}$fragmentation is visible in the calculation with rescaling alone as shown in Fig. 10. In addition, due to the smaller $K^{+}$-nucleus interaction cross section, the $K^{+}$ production on light nuclei may leave a chance to observe the pure rescaling effect. 

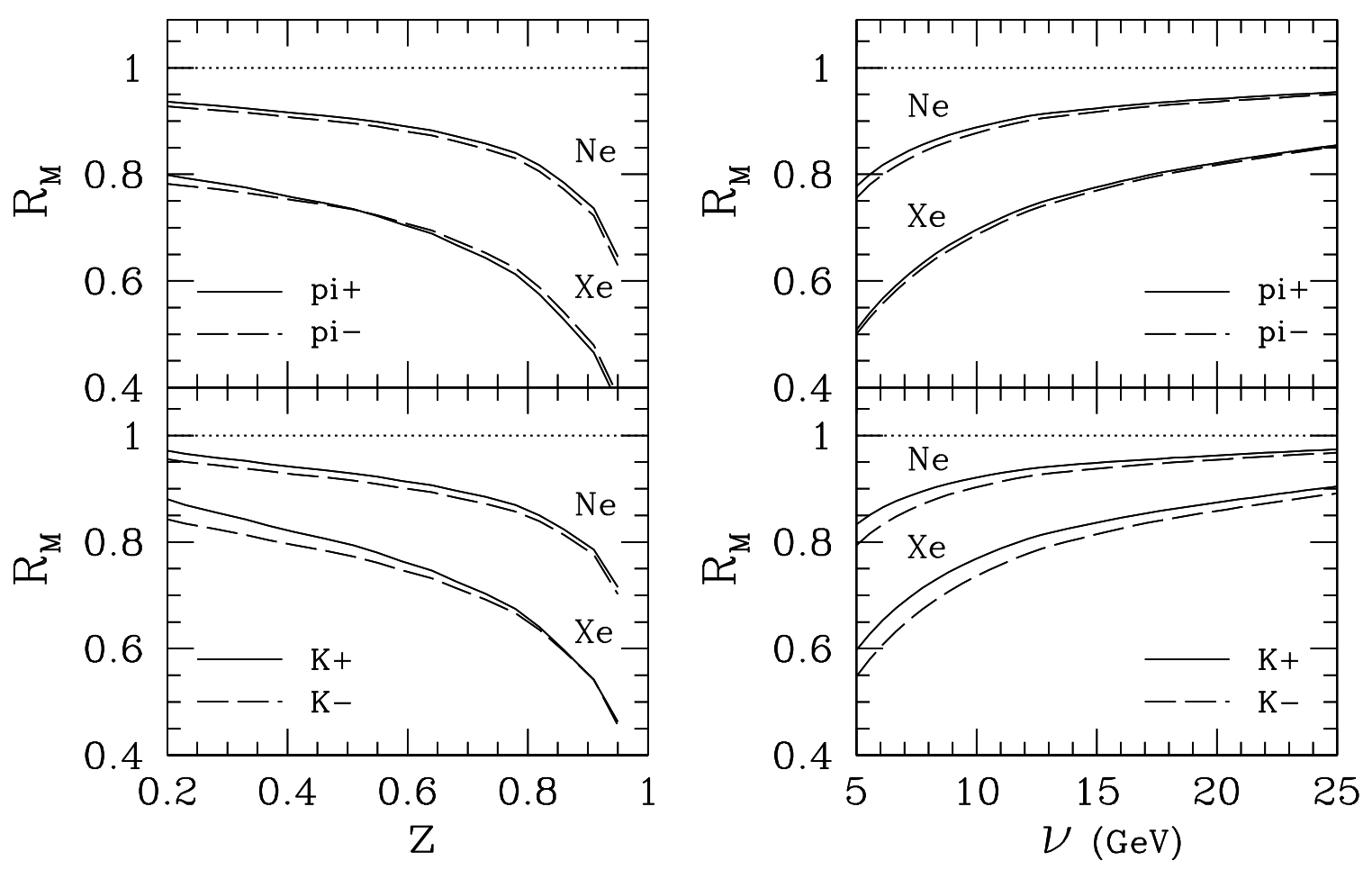

Figure 11: Predictions of the rescaling plus absorption effects at HERMES for charge- and flavourseparated multiplicity ratios on Neon and Xenon targets.

\section{Discussion and Conclusions}

We have considered two effects to describe semi-inclusive hadron production in nuclei: gluon radiation and nuclear absorption. In the final theoretical result the two effects add and until now the experimental situation does not clearly identify the rescaling effect. Theoretically, the gluon radiation occurs through the whole hadronization process even outside of the nucleus and criticism may be raised whether the rescaling correction is inappropriate in this case. We do not believe so. Rescaling extends the gluon window in $Q^{2}$ toward the infrared and includes thereby those gluons which are later absorbed by target fragments related to the nucleus. The fast forward gluons which go into the current fragmentation region have a higher time-like virtuality and are taken into account in the process of absorption of the prehadron. Therefore we think that no special necessity arises to truncate the rescaling correction due to formation outside of the nucleus. Of course, by assuming only a constant rescaling factor over the whole nucleus, we do not consider the escape of quarks in surface nucleons directly into the vacuum. In this case a more sophisticated treatment may be needed.

We have shown that rescaling models together with nuclear absorption are able to describe both HERMES and EMC data on the nuclear modification of hadron production in DIS process. While maximal deconfinement is ruled out by the data - it assumes a too large deconfinement - partial deconfinement is shown to have a possible effect on fragmentation functions. Nuclear absorption of the preformed hadron is shown to significantly affect the hadron production on heavy nuclei in the kinematic region of the HERMES experiments. The gluon-bremsstrahlung model of Ref. [16], that shares some similarities 
with the "outside-inside" picture of the Lund model, is also in good agreement with data at $z>0.5$. Further precise data at moderate and high $\nu$ 's and for light and heavy targets are needed to disentangle rescaling and formation length effects and to establish rescaling as an independent mechanism on its own.

Different scenarios for the space-time development of hadronization may be usefully tested against HERMES data. In our theoretical description they enter through the probability distributions $\mathcal{P}_{*}$, and eventually $\mathcal{P}_{h}$ if one separates between prehadron and hadron, and through the corresponding formation lengths $\left\langle l_{*}\right\rangle=\left\langle l_{F}\right\rangle$ and $\left\langle l_{h}\right\rangle=\left\langle l_{F}\right\rangle+z \nu / \kappa_{A}$. It would be interesting to measure $z$-distributions with low- and high- $\nu$ cuts in order to study how much the prehadronic absorption differs from hadronic absorption as the experimental data on $K^{-}$may suggest. This would allow to change the characteristic length scale $L=\nu / \kappa_{A}$ of the hadronization process. The high- $\nu$ data sample would be sensitive only on prehadron absorption, because the hadron is formed almost entirely outside the nucleus. On the other hand, in the low- $\nu$ data sample the information obtained on the prehadron absorption may be used to pin down the hadron absorption. Furthermore, the use of different cuts on $z$ would allow to map out the $\nu$-dependence of the absorption process more neatly. The necessity to have very accurate data in a wide range of $\nu$ and with different nuclei has also been stressed in the recommendation letter for a new European electron facility [39].

Acknowledgments. We are grateful to N. Bianchi, P. di Nezza, M.Gyulassy, J. Hüfner, B. Kopeliovich, A. Metz and P. Mulders for stimulating discussions. This work is partially funded by the European Commission IHP program under contract HPRN-CT-2000-00130. 


\section{Appendices}

\section{A EMC and HERMES acceptance}

In Table 3 we give the list of the EMC and HERMES kinematic range and experimental cuts, used in the numerical computations of this paper. The HERMES kinematic acceptance in $z$ and $\nu$ for pions and kaons is shown in Fig. 12. This figure explains the step structures of the LO computation of $\langle\nu\rangle(z)$ and $\langle z\rangle(\nu)$ shown in Fig. 8.

\begin{tabular}{ll||l|l||l|l||}
\multicolumn{1}{l||}{} & \multicolumn{1}{l||}{} & \multicolumn{2}{c||}{ EMC } & \multicolumn{2}{c||}{ HERMES } \\
\hline \multicolumn{1}{l||}{} & \multicolumn{1}{l||}{} & \multicolumn{2}{c||}{$h$} & $h$ & $\pi, K$ \\
\hline$E_{\text {beam }}$ & $\mathrm{GeV}$ & 100 & 200 & 27.5 & 27.5 \\
$Q_{\min }^{2}$ & $\mathrm{GeV}^{2}$ & 2 & 2 & 1 & 1 \\
$W_{\min }^{2}$ & $\mathrm{GeV}^{2}$ & 4 & 4 & 4 & 4 \\
$y_{\max }$ & & 0.85 & 0.85 & 0.85 & 0.85 \\
\hline$x_{\min }$ & & 0.02 & 0.02 & 0.06 & 0.02 \\
$x_{\max }$ & & 1 & 1 & 1 & 1 \\
$z_{\min }$ & & 0.2 & 0.2 & 0.2 & 0.2 \\
$z_{\max }$ & & 1 & 1 & 1 & 1 \\
$\nu_{\min }$ & $\mathrm{GeV}$ & 10 & 30 & 7 & 7 \\
$\nu_{\max }$ & $\mathrm{GeV}$ & 85 & 170 & 23.4 & 23.4 \\
$E_{h \min }$ & $\mathrm{GeV}$ & 3 & 3 & 1.4 & 2.5 \\
$E_{h \max }$ & $\mathrm{GeV}$ & 85 & 170 & 23.4 & 15.0 \\
\hline
\end{tabular}

Table 3: Kinematic cuts of the EMC and HERMES experiments.

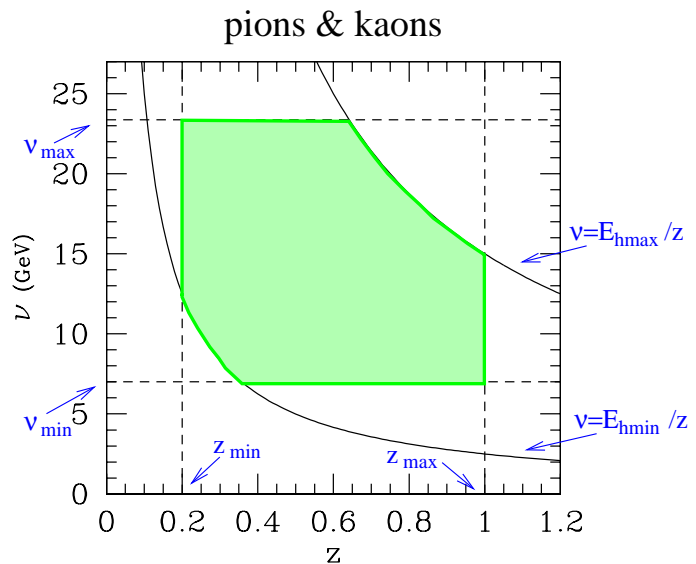

Figure 12: The HERMES kinematic acceptance in $z$ and $\nu$ for pions and kaons. 


\section{B Lund formation lengths}

We review the probability distribution of the formation lengths of a hadron with fractional momentum $z$ in the Lund model [12]:

$$
\mathcal{P}_{h}(y ; z, L)=\frac{\sum_{n=1}^{\infty} D_{n}(y ; z, L)}{\sum_{n=1}^{\infty} \int d y D_{n}(y ; z, L)},
$$

where $D_{n}(y ; z, L)$ is the longitudinal phase space density for the $n$-th rank hadron with fractional momentum $z$ to be formed at position $y$. For a prehadron with fractional momentum $z$, the probability distribution of the formation length is:

$$
\mathcal{P}_{*}(y ; z, L)=\mathcal{P}_{h}(y+z L ; z, L) .
$$

Consider Fig. 4 . The $n$-th pair production point $C_{n}$ has the light cone coordinates $y_{n}^{ \pm}=t_{n} \pm y_{n}$. The $n$-th rank hadron has light-cone momenta $p_{n}^{ \pm}=E_{n} \pm p_{n}$, and the fractional momenta are:

$$
z_{n}=\frac{E_{n}}{E_{0}}=\frac{p_{n}^{+}}{p_{0}^{+}} .
$$

The first constituent of the hadron $h_{n}$, formed in $C_{n}$, accelerates to the right under a constant string tension $\kappa_{A}$. At $P_{n}$, it has a light-cone momentum $p^{+}=2 \kappa_{A}\left(y_{n-1}-y_{n}\right)$. The second constituent of $h_{n}$, created in $C_{n-1}$, accelerates to the left, and at $P_{n}$ it has $p^{+}=0$.

$$
p_{n}^{+}=\kappa_{A}\left(y_{n-1}^{+}-y_{n}^{+}\right)
$$

The hadron fractional momentum $z_{n}$ for $n \geq 1$ is determined by the coordinates of the pair creation points $\left(\right.$ recall $\kappa_{A} /(2 \nu)=1 /(2 L)$ and $y_{0}^{+}=2 L, z_{0}=1$ ):

$$
z_{n}=\frac{y_{n-1}^{+}-y_{n}^{+}}{2 L} \text {. }
$$

The probability that a quark fragments into $n$ hadrons with fractional momenta $z_{1}, z_{2}, \ldots z_{n}$ is related to the normalized string fragmentation function $f(z)$ which gives the probability that a string breaks into two pieces carrying fractional momenta $z$ and $(1-z)$

$$
F\left(z_{1}, z_{2}, \ldots, z_{n}\right) \prod_{i=1}^{n} d z_{i}=\prod_{i=1}^{n} f\left(u_{i}\right) d u_{i}
$$

with relative fractional momenta $u_{i}$ :

$$
u_{n}=z_{n} \frac{1}{1-\sum_{k=1}^{n-1} z_{k}} .
$$

From Eq. (B.6) for $y_{i}^{+}$and using Eq. (B.7) we obtain:

$$
y_{n}^{+}=2 L\left(1-\sum_{k=1}^{n} z_{k}\right)=2 L \prod_{k=1}^{n}\left(1-u_{k}\right) \text {. }
$$


The longitudinal phase space densities $D_{1}(y ; z, L)$ of the first rank hadron and $D_{n}(y ; z, L)$ of the $n \geq 2$-th rank hadron to be formed at $y$ with fractional momentum $z$ are:

$$
\begin{aligned}
& D_{1}(y ; z, L)=\int d u_{1} f\left(u_{1}\right) \delta\left(z-z_{1}\right) \delta(y-L) \\
& D_{n}(y ; z, L)=\int \prod_{k=1}^{n} d u_{k} f\left(u_{k}\right) \delta\left(z-z_{n}\right) \delta\left(y-\frac{1}{2}\left[y_{n-1}^{+}-y_{n}^{-}\right]\right) .
\end{aligned}
$$

Since the $n$-th hadron production happens near the positive light-cone $y_{n}^{-} \approx 0$, we can use

$$
\delta\left(z-z_{n}\right) \delta\left(y-\frac{1}{2}\left[y_{n-1}^{+}-y_{n}^{-}\right]=\delta\left(z-u_{n} y / L\right) \delta\left(y-L \prod_{k=1}^{n-1}\left(1-u_{k}\right)\right)\right.
$$

to integrate $D_{n}$ over $u_{n}$ in Eq. (B.9). We obtain:

$$
D_{n}(y ; z, L)=\frac{L}{y} f\left(\frac{z L}{y}\right) \rho_{n}(y, L)
$$

where

$$
\begin{aligned}
& \rho_{1}(y ; L)=\delta(y-L) \\
& \rho_{n}(y ; L)=\int \prod_{k=1}^{n} d u_{k} f\left(u_{k}\right) \delta\left(y-L \prod_{k=1}^{n-1}\left(1-u_{i}\right)\right)
\end{aligned}
$$

are the densities of formation points of the $n$ th-rank hadron. The total density of hadron formation points is:

$$
\rho_{h}(y ; L)=\sum_{n=1}^{\infty} \rho_{n}(y, L)=\delta(y-L)+\sum_{n=2}^{\infty} \rho_{n}(y, L) .
$$

Consider now the string fragmentation function of the Lund model [29]:

$$
f(z)=\sum_{q} p_{q}\left(1+C_{q}\right)(1-z)^{C_{q}}
$$

where $p_{q}$ is a probability that a given production point involves pair production of a particular flavor $q$, and $C_{q}$ are parameters controlling the typical momentum fraction carried by final hadrons containing that flavor:

$$
C_{q}=\left\{\begin{array}{ll}
C & q=u, d, s \\
D_{q} & q \neq u, d, s
\end{array},\right.
$$

In the standard Lund model $C \approx 0.3$ and $D_{q}$ is flavour dependent. Note that the subscript $q$ includes not only quark flavours, but also diquarks in the fragmentation of the string into baryons. Since $p_{q} \ll 1$ for non light flavours $q \neq u, d, s$, we obtain from Eq. (B.12) and Eq. (B.13):

$$
\rho_{h}(y, L) \approx \delta(y-L)+\frac{1+C}{y}
$$


Finally, we obtain the probability distributions of the formation length of the prehadronic state, which for a flavour $q=u, d, s$ has $C_{q}=C$ and for $q \neq u, d, s$ has $C_{q}=D_{q}$ :

$$
\begin{aligned}
\mathcal{P}_{*}^{q}(y ; z, L)= & \frac{1+C_{q}}{(1+C)\left(1+z \frac{C q-C}{1+C_{q}}\right)} \frac{z L}{y-z L}\left[\frac{y}{(y+z L)(1-z)}\right]^{C_{q}} \\
& \times\left\{\delta[y-(1-z) L]+\frac{1+C}{y-z L} \theta[(1-z) L-y]\right\} \theta[y] .
\end{aligned}
$$

Note that the $\delta$-function represents the contribution from rank- 1 hadrons. The average formation length for the prehadronic state is then computed as $\left\langle l_{*}\right\rangle(z, L)=\int d y y \mathcal{P}_{*}(y ; z, L)$ :

$$
\begin{aligned}
\left\langle l_{*}\right\rangle= & \frac{1+C_{q}}{1+C+\left(C_{q}-C\right) z} \\
& \times\left[1+\frac{1+C}{2+C_{q}} \frac{(1-z)}{z^{2+C_{q}}}{ }_{2} F_{1}\left(2+C_{q}, 2+C_{q} ; 3+C_{q} ; \frac{z-1}{z}\right)\right](1-z) z L,
\end{aligned}
$$

where ${ }_{2} F_{1}$ is the Gauss' hypergeometric function [32]. Subtracting 1 from the square brackets in Eq. (B.16) would give the average formation length for hadrons of rank $n \geq 2$.

For a valence quark, $q=u, d, s$, the above expression reduces to Eq. (3.4), which is the one used in this paper. On the other hand, using Eq. (B.16) with $C_{q}=1$ and $C=0$ as considered in Ref. [34], the prehadron formation length is

$$
\left\langle l_{*}\right\rangle=\left[\frac{\ln \left(1 / z^{2}\right)-1+z^{2}}{1-z^{2}}\right] z L
$$

which is larger than in the standard Lund model as shown in Fig. 5.

\section{Absorption formulae with two length scales}

When considering a different cross-section for the hadron and the prehadron interaction with the nucleons, $\sigma_{*} \neq \sigma_{h}$, equations (4.4) and (4.6) have to be modified to take into account the propagation of the prehadronic state. In the Lund model considered in this paper, it propagates through a fixed length equal to $z \nu / \kappa_{A}$ before the formation of the hadron.

In the Bialas-Gyulassy model Eq. (4.4) becomes

$$
W_{0}\left(y^{\prime}, z, \nu\right)=1-\sigma_{*} \int_{y^{\prime}}^{y^{\prime}+z \nu / \kappa_{A}} d y_{*} \rho_{A}\left(b, y_{*}\right)-\sigma_{h} \int_{y^{\prime}+z \nu / \kappa_{A}}^{\infty} d y_{*} \rho_{A}\left(b, y_{*}\right) .
$$

In the Bialas-Chmaj model, the two-stage fragmentation process is modeled as a chain decay with two average decay lengths: $\left\langle l_{*}\right\rangle=\left\langle l_{F}\right\rangle$ for the "decay" of the quark into the prehadronic state, and $\langle\Delta l\rangle=z \nu / \kappa$ for the "decay" of the prehadronic state into the observed hadron $\left(\left\langle l_{F}\right\rangle\right.$ is given in Eq. (3.4)). Eq. (4.6) becomes then

$$
S_{A}(b, y)=1-\sigma_{*} \int_{y}^{\infty} d y^{\prime} P_{*}\left(y^{\prime}-y\right) \rho_{A}\left(b, y^{\prime}\right)-\sigma_{h} \int_{y}^{\infty} d y^{\prime} P_{h}\left(y^{\prime}-y\right) \rho_{A}\left(b, y^{\prime}\right),
$$


where $P_{*}\left(y^{\prime}-y\right)$ and $P_{h}\left(y^{\prime}-y\right)$ are, respectively, the probability of survival of a prehadron and a hadron at a distance $y^{\prime}-y$ from the virtual photon interaction point. When $\left\langle l_{*}\right\rangle \neq\langle\Delta l\rangle$ one has:

$$
\begin{aligned}
& P_{*}\left(y^{\prime}-y\right)=\frac{\langle\Delta l\rangle}{\left\langle l_{*}\right\rangle-\langle\Delta l\rangle}\left(e^{-\left(y^{\prime}-y\right) /\left\langle l_{*}\right\rangle}-e^{-\left(y^{\prime}-y\right) /\langle\Delta l\rangle}\right), \\
& P_{h}\left(y^{\prime}-y\right)=1-e^{-\left(y^{\prime}-y\right) /\langle l *\rangle}-P_{*}\left(y^{\prime}-y\right) .
\end{aligned}
$$

When $\left\langle l_{*}\right\rangle=\langle\Delta l\rangle$ the prehadron survival probability is:

$$
P_{*}\left(y^{\prime}-y\right)=\frac{1}{\left\langle l_{*}\right\rangle}\left(y^{\prime}-y\right) e^{-\left(y^{\prime}-y\right) /\left\langle l_{*}\right\rangle} .
$$




\section{References}

[1] A. Airapetian et al. [HERMES Collaboration], Eur. Phys. J. C 21 (2001) 599.

[2] J. Ashman et al. [European Muon Collaboration], Z. Phys. C 52 (1991) 1.

[3] A. Airapetian et al. [HERMES Collaboration], Eur. Phys. J. C 20 (2001) 479; V. Muccifora [HERMES Collaboration], arXiv:hep-ex/0106088.

[4] L. S. Osborne et al., Phys. Rev. Lett. 40 (1978) 1624.

[5] R. Baier, Yu.L. Dokshitzer, A.H. Mueller, D. Schiff, Nucl. Phys. B 484 (1997) 403; B.G.Zakharov, JETP Lett. 63 (1996) 952; M. Gyulassy, P. Levai, I. Vitev Nucl. Phys. B 594 (2001) 371; U.A. Wiedemann, Nucl. Phys. A 698 (2002) 615.

[6] X. N. Wang, Z. Huang and I. Sarcevic, Phys. Rev. Lett. 77 (1996) 231; X. N. Wang and Z. Huang, Phys. Rev. C 55 (1997) 3047.

[7] K. Adcox et al. [PHENIX Collaboration], Phys. Rev. Lett. 88 (2002) 022301.

[8] O. Nachtmann and H. J. Pirner, Z. Phys. C 21 (1984) 277.

[9] R. L. Jaffe, F. E. Close, R. G. Roberts and G. G. Ross, Phys. Lett. B 134 (1984) 449; Phys. Rev. D 31 (1985) 1004.

[10] O. Nachtmann and H. J. Pirner, Annalen Phys. 44 (1987) 13.

[11] J. Dias de Deus, Phys. Lett. B 166 (1986) 98.

[12] A. Bialas and M. Gyulassy, Nucl. Phys. B 291 (1987) 793.

[13] A. Bialas and T. Chmaj, Phys. Lett. B 133 (1983) 241.

[14] J. Czyzewski and P. Sawicki, Z. Phys. C 56 (1992) 493.

[15] J. Czyzewski, Acta Phys. Polon. B 21 (1990) 41.

[16] B. Kopeliovich, J. Nemchik and E. Predazzi, arXiv:nucl-th/9607036, published in proceedings of Workshop on Future Physics at HERA, Hamburg, Germany, 30-31 May 1996.

[17] X. F. Guo and X. N. Wang, Phys. Rev. Lett. 85 (2000) 3591; X. N. Wang and X. F. Guo, Nucl. Phys. A 696 (2001) 788; E. Wang and X. N. Wang, Phys. Rev. Lett. 89 (2002) 162301.

[18] F. Arleo, JHEP 11 (2002) 044 and arXiv:hep-ph/0210105.

[19] N. Z. Akopov, G. M. Elbakian and L. A. Grigoryan, arXiv:hep-ph/0205123.

[20] M. M. Sargsian et al. arXiv:nucl-th/0210025.

[21] M. M. Sargsian, S. Simula and M. I. Strikman, Phys. Rev. C 66 (2002) 024001. 
[22] I. Sick, Phys. Lett. B 157 (1985) 13; J. P. Chen et al., Phys. Rev. Lett. 66 (1991) 1283; J. Jourdan, Nucl. Phys. A 603 (1996) 117; G. Ricco, M. Anghinolfi, M. Ripani, S. Simula and M. Taiuti, Phys. Rev. C 57 (1998) 356; G. Ricco, M. Anghinolfi, M. Ripani, M. Taiuti and S. Simula, Few Body Syst. Suppl. 10 (1999) 423.

[23] S. Kretzer, Phys. Rev. D 62 (2000) 054001.

[24] M. E. Peskin and D. V. Schroeder, "An Introduction To Quantum Field Theory," Addison-Wesley, 1995.

[25] M. Gluck, E. Reya and A. Vogt, Eur. Phys. J. C5 (1998) 461.

[26] K. J. Eskola, V. J. Kolhinen and C. A. Salgado, Eur. Phys. J. C 9 (1999) 61.

[27] B. A. Kniehl, G. Kramer and B. Potter, Nucl. Phys. B 582 (2000) 514.

[28] X. F. Zhang, G. Fai and P. Levai, arXiv:hep-ph/0205008.

[29] B. Andersson et al. Phys. Rep. 97, (1983) 31.

[30] B. Andersson, "The Lund Model", Oxford University Press, 1998.

[31] A. Bialas and J. Czyzewski, Phys. Lett. B 222 (1989) 132.

[32] W.Magnus and F. Oberhettinger, Formeln und Sätze für die speziellen Funktionen der mathematischen Physik, Berlin-Göttingen-Heidelberg, 1948.

[33] B. Z. Kopeliovich and F. Niedermayer, Phys. Lett. B 151 (1985) 437.

[34] N. Pavel, Ph.D Thesis, University of Wuppertal, Mai 1989.

[35] R. V. Reid, Annals Phys. 50 (1968) 411.

[36] K. Hagiwara et al., Phys. Rev. D 66 (2002) 010001.

[37] HERMES data available on: http://www-hermes.desy.de/notes/pub/trans-public-subject.html\#HADRONATTENUATION.

[38] A. Accardi and H. J. Pirner, Nucl. Phys. A 711 (2002) 260.

[39] Recommendation letter for a new European Electron Facility. NuPECC Report July 2001. 